\title{
Upregulation of microRNA-194-5p inhibits hypopharyngeal carcinoma cell proliferation, migration and invasion by targeting SMURF1 via the mTOR signaling pathway
}

\author{
SHAN XU, LIAN HUI, NING YANG, YAN WANG, NING ZHAO and XUE-JUN JIANG \\ Department of Ear, Nose and Throat, The First Hospital of \\ China Medical University, Shenyang, Liaoning 110001, P.R. China
}

Received May 17, 2018; Accepted November 23, 2018

DOI: $10.3892 /$ ijo.2019.4711

\begin{abstract}
Hypopharyngeal carcinoma (HPC) is an aggressive malignancy with the worst prognosis among all head and neck cancers. MicroRNAs (miRNAs) are involved in the development of many human cancers, and may function as oncogenes or tumor suppressors. The present study aimed to evaluate the effects of miRNA (miR)-194-5p on the proliferation and invasion of HPC cells and to identify the potential regulatory mechanism. First, miR-194-5p and Smad ubiquitin regulatory factor 1 (SMURF1) expression levels were examined in HPC tissues. Subsequently, to explore the effects of miR-194-5p on SMURF1, a dual-luciferase reporter gene assay was performed to verify the target relationship. To define the role of miR-194-5p in HPC progression, miR-194-5p upregulation and depletion were used to evaluate its effects on cell viability, invasion and migration. SMURF1 silencing and rapamycin [an inhibitor of the mammalian target of rapamycin (mTOR) signaling pathway] treatment were also used to analyze the regulatory mechanism in HPC. Finally, tumor growth was assessed in xenografted tumors in nude mice. SMURF1 was demonstrated to be highly expressed, whereas miR-194-5p was poorly expressed in HPC tissues; SMURF1 was identified as a target gene of miR-194-5p. FaDu hypopharyngeal squamous cell carcinoma cells treated with miR-194-5p mimics exhibited decreased viability, invasion and migration. The results indicated that miR-194-5p may inactivate the mTOR signaling pathway by targeting SMURF1. In addition, the in vivo experiments further verified these regulatory effects. These data suggested that miR-194-5p-targeted SMURF1 inhibition
\end{abstract}

Correspondence to: Dr Xue-Jun Jiang, Department of Ear, Nose and Throat, The First Hospital of China Medical University, 155 Nanjing North Street, Shenyang, Liaoning 110001, P.R. China E-mail: djiangxj@163.com

Key words: microRNA-194-5p, hypopharyngeal carcinoma, Smad ubiquitin regulatory factor 1 , mammalian target of rapamycin signaling pathway, proliferation, metastasis may be involved in the disruption of HPC progression through the repression of the mTOR signaling pathway.

\section{Introduction}

Hypopharyngeal carcinoma (HPC) is one of the most common head and neck squamous cell cancers; it is a relatively rare but heterogeneous malignancy with a poor prognosis, and hypopharyngeal squamous cell carcinoma (HSCC) is the most frequent type of HPC (1). HPC is mainly initiated in the pyriform sinus and the posterior wall of the hypopharynx (2). Organ-preserving chemoradiotherapy has been proposed as a practical choice for early stage HPC, with surgical resection and reconstruction reserved for patients with advanced and relapsed tumors (3). Despite recent advances in surgery and chemotherapy, 5-year survival rates for patients diagnosed with HPC have shown no significant improvement (4). Previous studies have suggested that microRNAs (miRNAs), a large subgroup of short non-coding RNAs, are aberrantly expressed in a wide range of human cancers, and serve significant roles in the initiation, progression and metastasis of various cancers, including head and neck squamous cell carcinoma $(5,6)$. The role of miRNAs in human cancer has gained much attention (7), but how these miRNAs may be involved in the onset and development of HPC has yet to be elucidated.

miRNA (miR)-194-5p may serve as a diagnostic indicator for myelodysplastic syndromes, in which downregulated miR-194-5p expression levels were reported to be responsible for the poor survival of patients with myelodysplastic syndromes (8). Additionally, miR-194-5p downregulation is correlated with drug resistance in metastatic renal cell carcinoma(9).Notably, the online prediction website TargetScan (http://www.targetscan.org/vert_71), used in the present study, predicted that miR-194-5p may target Smad ubiquitin regulatory factor 1 (SMURF1). SMURF1 is homologous to the E6-associated protein C-terminus (HECT) domain ubiquitin ligase, which is involved in several biological pathways that mediate ubiquitination and degradation (10). SMURF1 silencing promotes the reduction of the cancer stem cell-like population in head and neck squamous cell carcinoma (11). Furthermore, SMURF1 is reported to mediate the activation of the phosphatidylinositol-3-kinase (PI3K)/protein kinase B 
(AKT)/mammalian target of rapamycin (mTOR) pathway in clear cell renal cell carcinoma (12). mTOR, which is commonly deregulated in human cancers, has been reported to play pivotal roles in cell-signaling pathways (13). A previous study found that the inhibition of the mTOR/p70S6 kinase pathway could serve as a potential target for the treatment of esophageal squamous cell carcinoma owing to its activation in this disorder (14). On the basis of these findings, the current study put forth a hypothesis that miR-194-5p may serve as a tumor suppressor for HPC by regulating SMURF1, with the involvement of the mTOR signaling pathway. To test this hypothesis, the upregulation and depletion of miR-194-5p were used to determine the effects of miR-194-5p on HPC cell viability, invasion and migration, and SMURF1 silencing, together with mTOR inhibition, was introduced to investigate the potential regulatory mechanisms.

\section{Materials and methods}

Ethics statement. The present study was approved by the Ethics Committee of The First Hospital of China Medical University (Shenyang, China), and written informed consent was obtained from each participant prior to enrollment. The animal experiments were conducted with approval of the Animal Ethics Committee of The First Hospital of China Medical University, and all animal experimental procedures conformed to the provisions of the Guide for the Care and Use of Laboratory Animals by US Department of Agriculture (15).

Study subjects. A total of 30 patients that were pathologically diagnosed with HPC, and who underwent surgical resection at The First Hospital of China Medical University between June 2011 and December 2017, were enrolled into the present study. The enrolled subjects included 28 males and 2 females, with a mean age of $63.1 \pm 8.3$ years. None of the included patients underwent chemotherapy or radiotherapy prior to enrollment. HPC tissues and adjacent tissues (at least $2 \mathrm{~cm}$ away from the edge of cancer tissues, with no cancer cells identified under a microscope) were collected from these patients. All tissue specimens were fixed in $4 \%$ paraformaldehyde, embedded and sectioned, as described below. The age, sex, primary tumor range $(\mathrm{T})$ staging and regional lymph node metastasis $(\mathrm{N})$ staging of patients were recorded (Table I).

Hematoxylin and eosin $(H \& E)$ staining. HPC and adjacent tissues were fixed in $10 \%$ neutral formalin for $16-18 \mathrm{~h}$ at room temperature, dehydrated in gradient ethanol $[50,70,80,95$, 100 (I) and $100 \%$ (II)] for 15 min, embedded in paraffin at $60^{\circ} \mathrm{C}$ for $30 \mathrm{~min}$ and sectioned $(5 \mu \mathrm{m})$. The tissue sections were dewaxed by xylene I and xylene II for $15 \mathrm{~min}$, followed by rehydration at gradient ethanol $(100,95,80,70$ and $50 \%)$ for $5 \mathrm{~min}$, respectively, and then washed by distilled water for $1 \mathrm{~min}$. The sections were stained for $5 \mathrm{~min}$ with hematoxylin, washed with water for $1 \mathrm{~min}$, differentiated with $1 \%$ hydrochloric acid ethanol for $15 \mathrm{sec}$ and washed again with tap water for $15 \mathrm{~min}$. Subsequently, the sections were stained with $1 \%$ eosin for 3 min and washed with tap water for $1 \mathrm{~min}$. The sections were dehydrated by gradient ethanol and then cleared with xylene I and xylene II for $5 \mathrm{~min}$ each. Finally, the sections were sealed with neutral gum and observed under an optical microscope.

Immunohistochemical staining. The sections were dewaxed and rehydrated as aforementioned, followed by heat-induced antigen retrieval in a microwave at a power of $800 \mathrm{~W}$ at $90^{\circ} \mathrm{C}$ for $5 \mathrm{~min}$. Subsequently, the sections were incubated with $3 \% \mathrm{H}_{2} \mathrm{O}_{2}$ at room temperature for $10 \mathrm{~min}$, washed three times with PBS and blocked with 10\% goat serum (Gibco; Thermo Fisher Scientific, Inc., Waltham, MA, USA) for $10 \mathrm{~min}$ at room temperature. Sections were incubated with primary antibody against SMURF1 (1:100; catalog no. ab38866; Abcam, Cambridge, MA, USA) at $4^{\circ} \mathrm{C}$ overnight, and subsequently incubated with a horseradish peroxidase (HRP)-conjugated goat anti-rabbit secondary immunoglobulin (Ig)G antibody $\left(1: 2,000\right.$, catalog no. ab6721; Abcam) for $30 \mathrm{~min}$ at $37^{\circ} \mathrm{C}$. The sections were stained with diaminobenzidine at $37^{\circ} \mathrm{C}$ for $10 \mathrm{~min}$, counter-stained with hematoxylin for $30 \mathrm{sec}$ at $37^{\circ} \mathrm{C}$, differentiated with hydrochloric acid ethanol, dehydrated with gradient ethanol, cleared with xylene I and II, and sealed with neutral gum, as aforementioned. The slides were examined under an optical light microscope, and the positive cells were scored as previously described (16). According to the staining intensity and the percentage of positive cells, the cells with cytoplasm or membranes stained with brown or brown-yellow granules were considered as positive. A total of five visual fields were observed in each section with an optical microscope (magnification, x400), and 200 cells were counted in each field. The percentage of positive cells with brown/brown-yellow cytoplasm or with brown/brown-yellow granules was calculated. The staining intensity was scored as 3 points (dark brown), 2 points (brown-yellow), 1 point (light yellow) or 0 points (unclear or colorless); and the percentage of positive cells was graded as 3 points ( $>50 \%$ cells stained), 2 points ( $25-50 \%$ cells stained), 1 point ( $<25 \%$ cells stained) or 0 points (no cells stained). The staining results of the tumors were expressed as the product of the staining intensity and the percentage of positive cells, which were classified similarly, with $0-1$ for negative staining), 2-3 for weakly positive, $4-5$ for moderately positive), and $>5$ for strong positive.

Dual-luciferase reporter gene assay. The online target prediction website TargetScan (http://www.targetscan. org/vert_71) was used to determine whether miR-194-5p targeted SMURF1. The wild-type (wt) full length 3'-untranslated region (3'UTR) sequence of SMURF1 mRNA was amplified by polymerase chain reaction (PCR) and was ligated into the pGL3 dual-luciferase reporter gene vector (Promega Corporation, Madison, WI, USA) following XhoI and HindIII restriction enzyme digestion. Following site-directed mutagenesis of the potential complementary binding site, the pGL3 vector was double-enzyme digested by XhoI and HindIII and the mut fragment was inserted into the dual-luciferase reporter vector to construct mut recombinant dual luciferase reporter vector. A total of $200 \mathrm{ng}$ constructed vectors, $50 \mathrm{nmol} / 1 \mathrm{miR}-194-5 \mathrm{p}$ mimics (5'-UGCAGCAGCUUCTGCATGTCCT-3') or $50 \mathrm{nmol} / 1$ mimics-NC (Guangzhou RiboBio Co., Ltd., Guangzhou, China) and $5 \mu \mathrm{l}$ of Lipofectamine ${ }^{\circledR} 2000$ transfection reagents (Invitrogen; Thermo Fisher Scientific, Inc., Waltham, MA, 
Table I. Low miR-194-5p expression levels are associated with hypopharyngeal carcinoma.

\begin{tabular}{lcccc}
\hline & \multicolumn{3}{c}{$\begin{array}{c}\text { miR-194-5p } \\
\text { expression }\end{array}$} \\
\cline { 3 - 4 } $\begin{array}{l}\text { Clinicopathological } \\
\text { feature }\end{array}$ & $\mathrm{n}$ & Low & High & P-value \\
\hline Age (years) & 13 & 7 & 6 & 0.731 \\
$\quad$ 6 60 & 17 & 8 & 9 & \\
$>60$ & & & & 0.143 \\
Sex & 28 & 15 & 13 & \\
Male & 2 & 0 & 2 & \\
Female & & & & $\mathbf{0 . 0 4 6}$ \\
T stage & 9 & 2 & 7 & \\
T1+T2 & 21 & 13 & 8 & \\
T3+T4 & & & & $\mathbf{0 . 0 2 8}$ \\
N stage & 14 & 4 & 10 & \\
N0+N1 & 16 & 11 & 5 & \\
N2+N3 & & & & \\
\hline
\end{tabular}

a Data were evaluated by a $\chi^{2}$. miR, microRNA; $N$ stage, regional lymph node metastasis; $\mathrm{T}$ stage, primary tumor range. Bold print denotes statistical significance.

USA) were dissolved together in the $250 \mu$ l Opti-MEM culture solution (Invitrogen; Thermo Fisher Scientific, Inc.) to prepare the transfection mixture, according to the manufacturer's protocol, and let stand at room temperature for $20 \mathrm{~min}$. The transfection mixture was inoculated into 6-well plates, gently mixed with the cell culture solution by shaking and incubated in a $37^{\circ} \mathrm{C} \mathrm{CO}_{2}$ incubator. Following $6 \mathrm{~h}$ incubation, the culture medium and transfection reagents were replaced with $2 \mathrm{ml}$ of RPMI-1640 medium (Invitrogen; Thermo Fisher Scientific, Inc.) containing 10\% FBS (Gibco; Thermo Fisher Scientific, Inc.) for $72 \mathrm{~h}$ at $37^{\circ} \mathrm{C}$. Cells were collected and firefly and Renilla luciferase activities were analyzed with the Luciferase Reporter Gene Assay kit (Promega Corporation), according to the manufacturer's protocol; firefly luciferase activity was normalized to Renilla luciferase activity.

Reverse transcription-quantitative polymerase chain reaction $(R T-q P C R)$. Tissues $(100 \mathrm{mg})$ or cells $\left(5 \times 10^{6}\right)$ were used for total RNA extraction using TRIzol@ reagent (Invitrogen, Carlsbad, CA, USA), according to the manufacturer's protocol. cDNA was synthetized using the M-MLV Reverse Transcription kit (Invitrogen; Thermo Fisher Scientific, Inc.), according to the manufacturer's protocol; briefly, the reaction conditions were as follows: $37^{\circ} \mathrm{C}$ for $60 \mathrm{~min}$ and $99^{\circ} \mathrm{C}$ for $5 \mathrm{~min}$, and the reaction was terminated at $4^{\circ} \mathrm{C}$. The SYBR Prime Script miRNA RT-PCR kit (Takara Biotechnology Co., Ltd., Dalian, China) was used to determine the expressions of miR-194-5p in HPC and adjacent normal tissues, as well as the human HPC cell lines. The $20 \mu \mathrm{l}$ reaction was set up as follows: $10 \mu \mathrm{l}$ SYBR Premix Ex Taq II (2X), $0.8 \mu$ l of PCR forward primer (10 $\mu \mathrm{mol} / \mathrm{l}), 0.8 \mu \mathrm{l}$ of Universal-miR qPCR primer $(10 \mu \mathrm{mol} / \mathrm{l})$, $0.4 \mu 1$ of ROX Reference Dye II (50X), $2 \mu 1$ of cDNA template and $6 \mu \mathrm{l}$ of $\mathrm{ddH}_{2} \mathrm{O}$. The thermocycling conditions were as follows: Initial denaturation at $95^{\circ} \mathrm{C}(10 \mathrm{~min})$; followed by 40 cycles of denaturation at $95^{\circ} \mathrm{C}(10 \mathrm{sec})$, annealing at $60^{\circ} \mathrm{C}$ $(10 \mathrm{sec})$ and extension at $72^{\circ} \mathrm{C}(15 \mathrm{sec})$. The primers were synthesized by the Shanghai Sangon Biotechnology Co. Ltd. (Shanghai, China); sequences are indicated in Table II. U6 was used as an internal reference and for normalization of miR-194-5p, and GAPDH as an internal reference and for normalization for SMURF1; the relative expressions of miR-194-5p and SMURF1 were calculated using the $2^{-\Delta \Delta C q}$ method (17).

Western blot analysis. A total of $1 \times 10^{6}$ cells were collected and washed two times with PBS with the supernatant being discarded. Cells were lysed in radioimmunoprecipitation assay lysis buffer, followed by an ice bath at $4^{\circ} \mathrm{C}$ for $30 \mathrm{~min}$ and centrifugation at $25,764 \mathrm{x} \mathrm{g}$ for $15 \mathrm{~min}$. The supernatant was collected and transferred to another clean Eppendorf tube. Protein concentration was determined by a bicinchoninic acid assay. Protein samples (20-30 $\mu \mathrm{g})$ were separated by $12 \%$ SDS-PAGE. Proteins were transferred to a polyvinylidene fluoride membrane, which was subsequently blocked with 5\% skimmed milk in tris-buffered saline $+0.05 \%$ Tween-20 (TBST) on a shaker at room temperature for $30 \mathrm{~min}$. The membranes were incubated at room temperature for $2 \mathrm{~h}$ with primary rabbit polyclonal antibodies (diluted in blocking solution) against SMURF1 (1:500; catalog no. ab38866), mTOR $(1: 2,000$; catalog no. ab2732), phosphorylated (p)-mTOR (1:2,000; catalog no. ab109268), topoisomerase II (TOPO II; 1:2,000; catalog no. ab52934), minichromosome maintenance 2 (MCM2; 1:1,000; catalog no. ab108935), proliferating cell nuclear antigen (PCNA; 1:1,000; catalog no. ab18197), Ki67 (1:5,000; catalog no. ab92742), matrix metalloproteinase (MMP)-2 (1:2,000; catalog no. ab92536), MMP-9 (1:1,000; catalog no. ab38898) and GAPDH (1:2,500; catalog no. ab9485), all purchased from Abcam. The membranes were subsequently incubated with an HRP-conjugated secondary goat anti-rabbit IgG antibody (1:2,000; catalog no. ab6721; Abcam) at room temperature for $1 \mathrm{~h}$. Protein bands were visualized using Enhanced Chemiluminescence reagents (EMD Millipore, Billerica, MA, USA), and washed three times with TBST, 10 min each. Images were captured with a Gel Doc XR gel imaging instrument (Bio-Rad Laboratories, Inc., Hercules, CA, USA) and densitometric analysis was conducted using ImageJ software (version 1.42, National Institutes of Health, Bethesda, MD, USA); GAPDH was used as an internal control and for normalization.

Cell culture and transfection. Human HPC cell lines FaDu and HSC-4 were provided by the Cancer Institute of China Medical University (Beijing, China). Cells were subcultured in Dulbecco's modified Eagle's medium (DMEM; Gibco; Thermo Fisher Scientific, Inc.) containing $10 \%$ fetal bovine serum (FBS; Gibco; Thermo Fisher Scientific, Inc.) and 1\% streptomycin at $37^{\circ} \mathrm{C}$ with $5 \% \mathrm{CO}_{2}$ and saturated humidity. The medium was replaced every 2 days, and the cells that grew well were used for cell selection. The sequences of the transfected components are as follows: miRNA mimic-negative control (mimic-NC), 5'-ACATTGTCGTTGAGGTACAC 
Table II. Primer sequences for reverse transcription quantitative polymerase chain reaction.

\begin{tabular}{ll} 
Gene & \multicolumn{1}{c}{ Primer sequence (5'-3') } \\
\hline miR-194-5p & F: 5'-GCCGTCTGTAACAGCAACTCCA-3' \\
& R: 5'-GTGCAGGTCCGAGGTATTC-3' \\
SMURF1 & F: 5'-CTCATCCCTCAACATCTGCTG-3' \\
& R: 5'-GCCCTCCTTTCTTCATCG-3' \\
U6 & F: 5'-GCTTCGGCAGCACATATACT-3' \\
& R: 5'-GTGCAGGGTCCGAGGTATTC-3' \\
GAPDH & F: 5'-GAAGGTCGGAGTCAACGGAT-3' \\
& R: 5'-CCTGGAAGATGGTGATGGGAT-3'
\end{tabular}

F, forward; miR, microRNA; R, reverse; SMURF1, Smad ubiquitin regulatory factor 1 .

CT-3'; miR-194-5p inhibitor-NC, 5'-AGGUTCAACUTGA CGTACAGGA3'; miR-194-5p inhibitor, 5'-TCCACATGGAGT TGCTGTTACA-3'; and SMURF1 (XM_166483) small interfering (si)RNA 5'-AAGAACCUUGCAAAGAAAGAC-3'. Nine experimental groups were generated through liposome-mediated transfection and included: i) Control, FaDu cells without any treatment; ii) mimic-NC, FaDu cells transfected with mimic-NC; iii) miR-194-5p mimics, FaDu cells transfected with miR-194-5p mimics; iv) inhibitor-NC, $\mathrm{FaDu}$ cells transfected with inhibitor-NC; v) miR-194-5p inhibitor, FaDu cells transfected with miR-194-5p inhibitor; vi) miR-194-5p inhibitor + NC-siRNA, FaDu cells co-transfected with miR-194-5p inhibitor and NC siRNA; vii) miR-194-5p inhibitor + SMURF1 siRNA, FaDu cells transfected with miR-194-5p inhibitor + SMURF1 siRNA; viii) miR-194-5p inhibitor $+0.01 \%$ dimethyl sulfoxide (DMSO), FaDu cells transfected with miR-194-5p inhibitor and treated with DMS; ix) and miR-194-5p inhibitor + rapamycin, FaDu cells transfected with miR-194-5p inhibitor and treated with $100 \mathrm{ng} / \mathrm{ml}$ rapamycin. The mimics-NC, inhibitor-NC, NC-siRNA, miR-194-5p mimics, miR-194-5p inhibitor and SMURF1 siRNA were purchased from Guangzhou RiboBio Co., Ltd.; DMSO and rapamycin was obtained from Beijing Propbs Biotechnology Co., Ltd. (Beijing, China). Cells were seeded $\left(1 \times 10^{5}\right.$ cells/well) into a 6 -well plate; when the cells reached $\sim 30 \%$ confluency, a transfection mixture was prepared in accordance with the instructions of the Lipofectamine 2000 transfection reagent: $10 \mu \mathrm{miR}-194-5 \mathrm{p}$ mimics or $50 \mathrm{nmol} / \mathrm{l}$ inhibitor, mimics-NC, inhibitor-NC, NC-siRNA or SMURF1 siRNA and $5 \mu \mathrm{l}$ of Lipofectamine 2000 transfection reagents were dissolved together in the Opti-MEM (Invitrogen; Thermo Fisher Scientific, Inc.) culture solution to prepare the transfection mixture, and let stand at room temperature for $20 \mathrm{~min}$. The transfection mixture was inoculated into 6-well plates, gently mixed with the cell culture solution by shaking and incubated in a $37^{\circ} \mathrm{C} \mathrm{CO}_{2}$ incubator for $6 \mathrm{~h}$; after which, the culture medium and transfection reagents were replaced with $2 \mathrm{ml}$ of RPMI-1640 medium containing 10\% FBS and incubated for $24 \mathrm{~h}$ prior to subsequent experimentation.
Cell Counting Kit-8 (CCK-8). At 24 h post-transfection, $\mathrm{FaDu}$ cells were treated with $0.25 \%$ trypsin and collected. Cells were centrifuged at $200 \mathrm{x} \mathrm{g}$ for $5 \mathrm{~min}$ at $4^{\circ} \mathrm{C}$ and resuspended in fresh complete DMEM and inoculated into 96-well plates at a density of $0.8 \times 10^{3}$ cells/well (100 $\left.\mu \mathrm{l} / \mathrm{well}\right)$, with five duplicated wells for each group. A CCK-8 kit (Dojindo Molecular Technologies, Inc., Kumamoto, Japan) was used to examine cell viability. A total of $10 \mu \mathrm{l}$ CCK-8 solution was gently added into each well along the wall, gently shaken and mixed while avoiding bubbles. The mixture was incubated at $37^{\circ} \mathrm{C}$ for $2 \mathrm{~h}$, and the optical density was measured at a wavelength of $450 \mathrm{~nm}$ on an automatic enzyme immunoassay instrument after $0,24,48,72$ and $96 \mathrm{~h}$ of transfection, and the growth curve was plotted.

Transwell cell migration and invasion assay. Following $24 \mathrm{~h}$ transfection, FaDu cells were detached with trypsin, and single cell suspensions were prepared. Cells were washed two times with serum-free medium, and the number of cells was counted and the concentration was adjusted to $5 \times 10^{6}$ cells $/ \mathrm{ml}$. A total of $100 \mu 1$ of cell suspension was slowly added to the upper chamber of each Transwell chamber with or without Matrigel (for invasion and migration, respectively), and $600 \mu 1$ medium containing 10\% FBS was added to the lower chamber; a total of three duplicated wells were used for each group, and the mixture was cultured in a $5 \% \mathrm{CO}_{2}$ incubator at $37^{\circ} \mathrm{C}$ for $24 \mathrm{~h}$. The non-invading cells in the upper membrane were removed with cotton swabs, and cells in the lower chamber were fixed in methanol for $10 \mathrm{~min}$ and stained with $0.1 \%$ crystal violet for $40 \mathrm{~min}$. Five visual fields (magnification, x100) were randomly examined and the number of invading cells in each group was counted; the average value was recorded.

Xenograft tumors in nude mice. A total of 54 male athymic BALB/c nude mice (age, 4-5 weeks; weight $18 \pm 2 \mathrm{~g}$ ) were purchased from Beijing Vital River Laboratory Animal Technology Co., Ltd. (Beijing, China). Cells in the logarithmic growth phase collected and resuspended in PBS to a concentration of $1 \times 10^{7}$ cells $/ \mathrm{ml}$. A total of 54 nude mice were randomly divided into 9 groups ( $\mathrm{n}=6$ mice/group): i) Control group, which were injected with untreated cells; ii) mimics-NC group, which were injected with mimic-NC-transfected cells; iii) miR-194-5p mimics group, which were injected with miR-194-5p mimics-transfected cells; iv) inhibitor-NC group, which were injected with miR-194-5p inhibitor-NC-transfected cells; v) miR-194-5p inhibitor group, which were injected with miR-194-5p inhibitor-transfected cells; vi) miR-194-5p inhibitor + NC siRNA group, which were injected with cells co-transfected with miR-194-5p inhibitor and NC siRNA; vii) miR-194-5p inhibitor + SMURF1 siRNA group, which were injected with cells co-transfected with miR-194-5p inhibitor and SMURF1 siRNA; viii) miR-194-5p inhibitor + DMSO group, which were injected with miR-194-5p inhibitor-transfected cells that were co-treated with DMSO); xi) and miR-194-5p inhibitor + rapamycin group, which were injected with miR-194-5p mimics-transfected cells that were co-treated with rapamycin. The cell suspensions containing $1 \times 10^{7}$ transfected or treated cells were injected into the right armpit of the nude mice using a disposable 


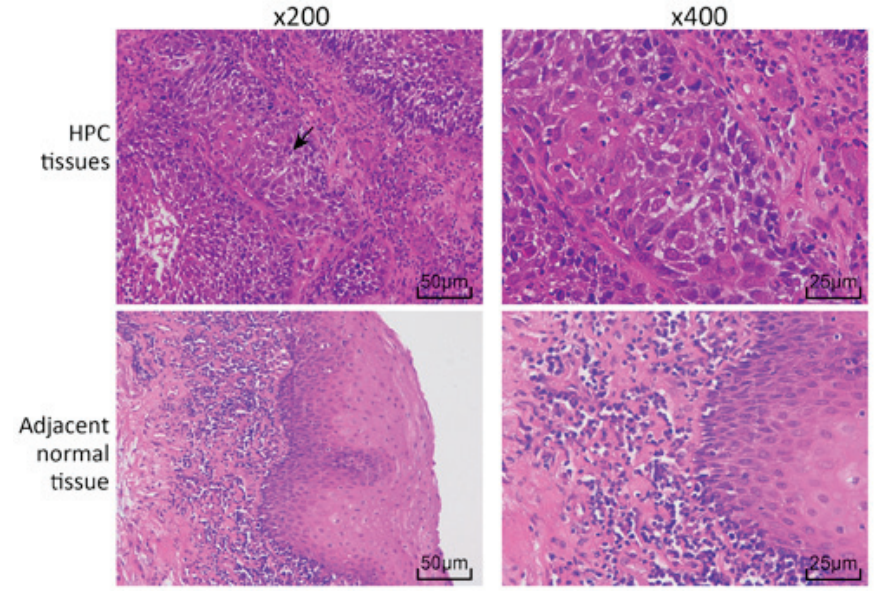

Figure 1. Histopathological examination of HPC tissues and adjacent norma tissues. HPC tissue cells were arranged in a disordered manner, with large nuclei that were deeply stained; cancer nests were observed in the epithelial cells (arrow). Cells in the adjacent normal tissues were arranged in an organized way. H\&E, hematoxylin-eosin; HPC, hypopharyngeal carcinoma.

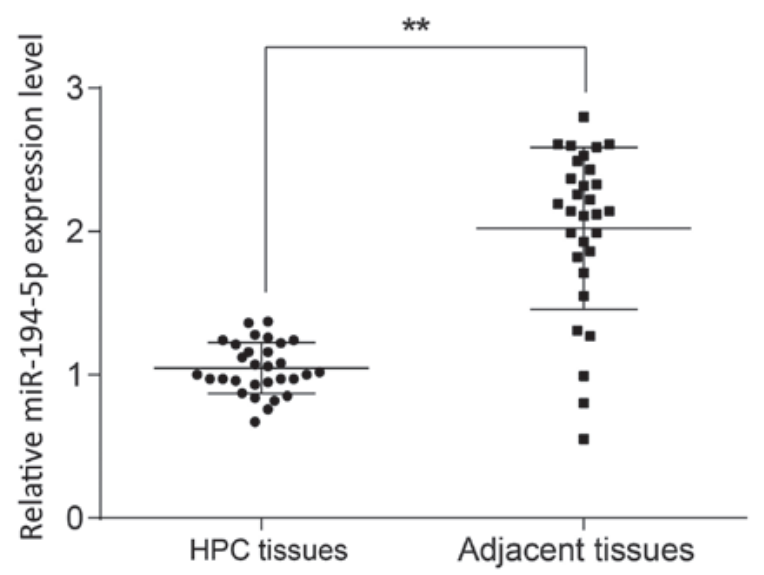

Figure 2. miR-194-5p expression was downregulated in HPC. The expression levels of miR-194-5p in HPC tissues and adjacent normal tissues detected by reverse transcription-quantitative polymerase chain reaction. The data are evaluated by paired $t$-test; ${ }^{* *} \mathrm{P}<0.01$. HPC, hypopharyngeal carcinoma; miR, microRNA.

syringe. Experiments were conducted in a specified pathogen-free barrier environment. Nude mice were raised until the tumor was visible with the naked eye. Tumor volume (volume $=\pi / 6 \mathrm{x}$ short diameter ${ }^{2} \mathrm{x}$ long diameter) of nude mice was measured on days 7, 14, 21 and 28 following tumor transplantation. The nude mice were sacrificed on day 28 , and the weight of the tumors was measured.

Statistical analysis. Statistical analyses were conducted using SPSS 21.0 software (IBM Corp., Armonk, NY, USA). Data are expressed as the mean \pm standard deviation. Comparisons between two groups were analyzed by independent-sample t-test or paired t-test. Comparisons of miR-194-5p and clinicopathological features between two groups were conducted using the $\chi^{2}$ test. The correlation between miR-194-5p and SMURF1 expressions was analyzed by Pearson's correlation analysis. Comparisons of data among multiple groups were tested using one-way analysis of variance (ANOVA) followed by
Tukey's post hoc test. Comparisons of cell viability and tumor volume at different time points were analyzed by two-way ANOVA. $\mathrm{P}<0.05$ was considered to indicate a statistically significant difference.

\section{Results}

Histopathological examination of HPC tissues and adjacent normal tissues. HPC and adjacent normal tissues were stained with $\mathrm{H} \& \mathrm{E}$ to examine the histopathological changes (Fig. 1). In the HPC tissues, cells were arranged in a disordered manner, with large nuclei that were deeply stained, cancer nests were observed in the epithelial cells. However, cells in the adjacent normal tissues were arranged in an organized way.

DownregulatedmiR-194-5pisassociated with HPCprogression. RT-qPCR was performed to examine the expression levels of miR-194-5p in HPC tissues, and the results demonstrated that miR-194-5p expression was significantly lower in HPC tissues compared with expression in adjacent normal tissues $(\mathrm{P}<0.01$; Fig. 2). The median value of miR-194-5p expression as set as the cut-off value, and the 30 HPC tissues were divided into a high expression group (miR-194-5p expression $\geq 1.02$ ) and a low expression group (miR-194-5p expression $<1.02$ ) to analyze the relationship between the expression level of miR-194-5p and the clinicopathological features in patients with HPC. The results indicated that there was no significant relationship between the expression of miR-194-5p and age or sex ( $>>0.05$; Table I), whereas low miR-194 expression levels were associated with $\mathrm{T} / \mathrm{N}$ stages. These results indicated that miR-194-5p expression was downregulated in HPC, which may be involved in the progression and metastasis of HPC.

Upregulated miR-194-5p reduces viability, migration and invasion of FaDu cells and inhibits tumor growth. The expression levels of miR-194-5p in the HPC cell lines FaDu and HSC-4 were examined using RT-qPCR. The results demonstrated that the expression of miR-194-5p in the HSC-4 cell line was significantly higher compared with expression in the FaDu cells $(\mathrm{P}<0.05$; Fig. 3A). Therefore, the FaDu cell line was selected for subsequent experimentation. FaDu cells were transfected with miR-194-5p mimics or inhibitor and successful transfections were confirmed by RT-qPCR, which demonstrated a significantly increased or reduced level of miR-194-5p expression, respectively, compared with the NC-transfected groups ( $\mathrm{P}<0.01$; Fig. 3B).

CCK-8 was used to assess the effects of miR-194-5p on the viability of FaDu cells. When the expression of miR-194-5p was upregulated, the viability of FaDu cells decreased significantly, whereas viability was significantly increased following the downregulation of miR-194-5p expression (both $\mathrm{P}<0.01$; Fig. 3C), which indicated that miR-194-5p may inhibit the viability of FaDu cells. In addition, the protein expression levels of the proliferation-related factors TOPO II, MCM2, PCNA and Ki67 were examined by western blot analysis following miR-194-5p mimics or inhibitor transfection, all of which were significantly decreased following miR-194-5p mimics transfections and significantly increased following transfection with the miR-194-5p inhibitor, compare with the respective controls ( $\mathrm{P}<0.01$; Fig. $3 \mathrm{D})$. 
A

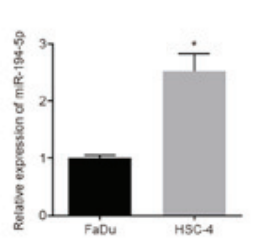

B

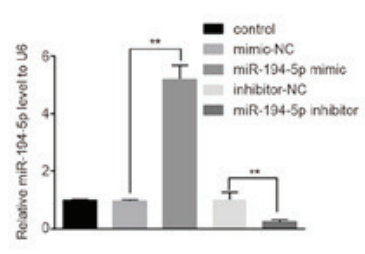

C

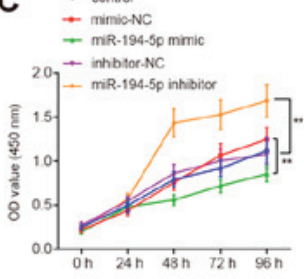

D

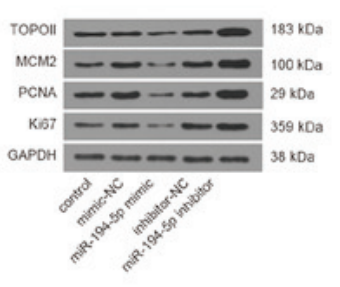

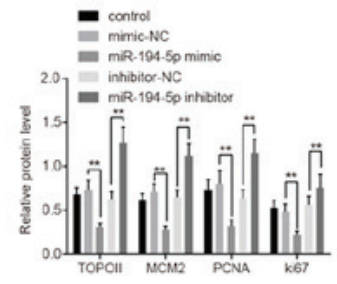
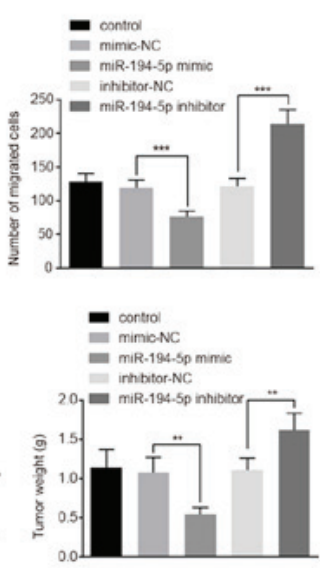

Figure 3. High miR-194-5p expression reduces FaDu cell viability, invasion and migration. (A) miR-194-5p expression in two HPC cell lines FaDu and HSC-4 detected by RT-qPCR. (B) miR-194-5p expression detected by RT-qPCR following miR-194-5p mimics or inhibitor transfection into FaDu cells (C) OD values were detected by Cell Counting Kit-8 at different time points following miR-194-5p mimics or inhibitor transfections. (D) Protein expression levels of proliferation-related genes TOPO II, MCM2, PCNA and Ki67 were determined by western blot analysis. (E) Invasive ability of transfected FaDu cells was measured by Matrigel assay; magnification, x400. (F) Migratory ability of transfected FaDu cells was measured by Transwell assay; magnification, x400. (G) Protein expression levels of invasion-related factors MMP-2 and MMP-9 were detected by western blot analysis. (H) Nude mice were injected with cells treated with miR-194-5p mimics or inhibitor, and tumor volumes and weights were examined. Experiments were repeated three times, and data are presented as the mean \pm standard deviation; ${ }^{*} \mathrm{P}<0.05 ;{ }^{* *} \mathrm{P}<0.01 ;{ }^{* * *} \mathrm{P}<0.001$. HPC, hypopharyngeal carcinoma; MCM2, minichromosome maintenance 2 ; miR, microRNA; MMP, matrix metalloproteinase; NC, negative control; OD, optical density; PCNA, proliferating cell nuclear antigen; RT-qPCR, reverse transcription-quantitative polymerase chain reaction; TOPO II, topoisomerase II.

The effects of miR-194-5p on the invasion and migration of FaDu cells was evaluated by Matrigel and Transwell assays, respectively. When the expression of miR-194-5p was upregulated, the number of invasive FaDu cells was significantly decreased, whereas the downregulation of miR-194-5p resulted in an increase in the number of invasive cells $(\mathrm{P}<0.01$; Fig. 3E); these results suggested that miR-194-5p may negatively regulate the invasive ability of FaDu cells. Similarly, miR-194-5p mimics transfection resulted in a significant decrease in the number of migrating FaDu cells, whereas the number of migrating cells were significantly increased following miR-194-5p inhibitor transfection $(\mathrm{P}<0.001$; Fig. $3 \mathrm{~F})$; these data suggested that high expression levels of miR-194-5p may inhibit the migratory ability of $\mathrm{FaDu}$ cells. In addition, the expression of invasion-related factors MMP-2 and MMP-9 were detected by western blot analysis following transfection, and the results demonstrated that the protein expression levels of MMP-2 and MMP-9 were significantly decreased by miR-194-5p upregulation and significantly increased by miR-194-5p depletion $(\mathrm{P}<0.01$; Fig. 3G).

Finally, the data described above were verified with in vivo experiments by means of the xenograft tumors in nude mice (Fig. 3H). Compared with the inhibitor-NC group, tumor volume in the nude mice transplanted with the miR-194-5p inhibitor-treated cells was increased, and the weight of tumors after 28 days was also significantly increased. Compared with the mimics-NC group, tumor volume in the nude mice was reduced and the tumor weight after 28 days was significantly decreased in the miR-194-5p mimics group $(\mathrm{P}<0.05)$. These in vivo experimental results indicated that elevated miR-194-5p expression levels may contribute to the inhibition of tumor growth.

miR-194-5p binds to the SMURF1 3'UTR. miR-194-5 target genes were predicted using the TargetScan online prediction website, which indicated that the seed sequence of miR-194-5p targets the 3'UTR of SMURF1 mRNA (Fig. 4A). This potential interaction was examined using luciferase assays in FaDu cells co-transfected with either SMURF1-wtUTR or SMURF1-mutUTR and miR-194-5p mimics. The luciferase activity of FaDu cells was significantly decreased in SMURF1-wtUTR and miR-194-5p mimics co-treated cells $(\mathrm{P}<0.01$; Fig. 4B), which further demonstrated that miR-194-5p can bind to and regulate SMURF1 expression. Pearson's correlation analysis was used to verify the correlation between miR-194-5p and SMURF1 mRNA, the results of which indicated a negative correlation between SMURF1 and miR-194-5p expression ( $\mathrm{r}=-0.480 ; \mathrm{P}<0.01$; Fig. 4C). Subsequently, immunohistochemical staining was performed to determine the expression of SMURF1 in human HPC tissues and adjacent tissues, which demonstrated that SMURF1 was mainly expressed in the cytoplasm and cell membrane (Fig. 4D). The positive rate of SMURF1 protein 
A

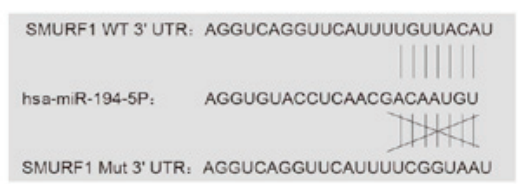

B

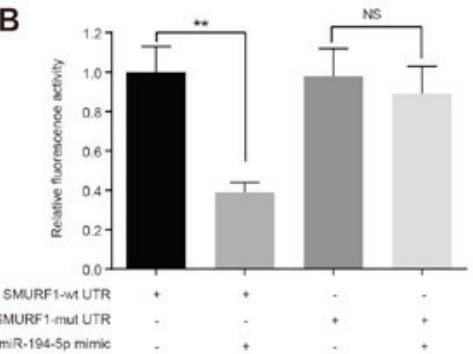

C

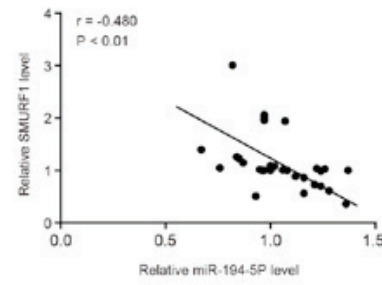

D

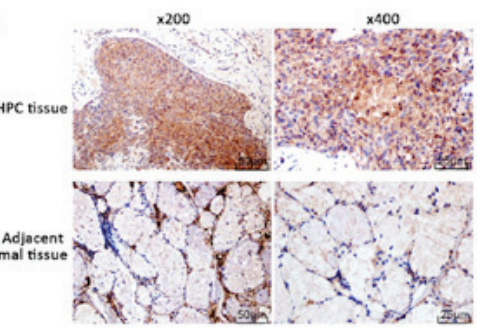

$E$

F
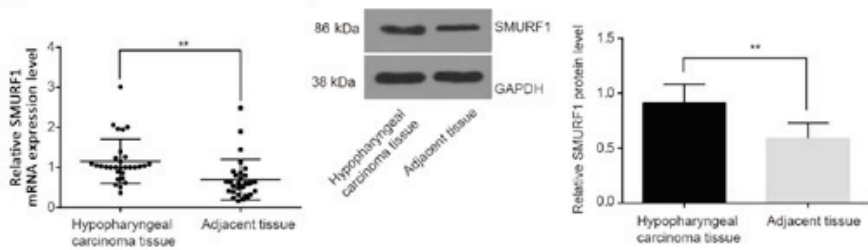

Figure 4. SMURF1 is overexpressed in HPC tissues and is a target gene of miR-194-5p. (A) miR-194-5p target sites in the SMURF1-wt 3'-UTR were predicted using the TargetScan online prediction website. (B) The dual-luciferase reporter gene assay was used to verify that SMURF1 is a target gene of miR-194-5p. (C) Correlation between SMURF1 and miR-194-5p expressions was assessed using Pearson's correlation analysis. (D) SMURF1 protein expression in HPC and normal adjacent tissues was detected by immunohistochemical staining; $n=30$. (E) mRNA expression levels of SMURF1 in HPC tissues and adjacent tissues were determined by reverse transcription-quantitative polymerase chain reaction; $n=30$. (F) SMURF1 protein expression levels in HPC and normal adjacent tissues were determined by western blot analysis. Experiments were repeated three times, and data are presented as the mean \pm standard deviation; ${ }^{* *} \mathrm{P}<0.01$. HPC, hypopharyngeal carcinoma; miR, microRNA; mut, mutant; NS, no statistical significance; SMURF1, Smad ubiquitin regulatory factor 1; wt, wild-type.
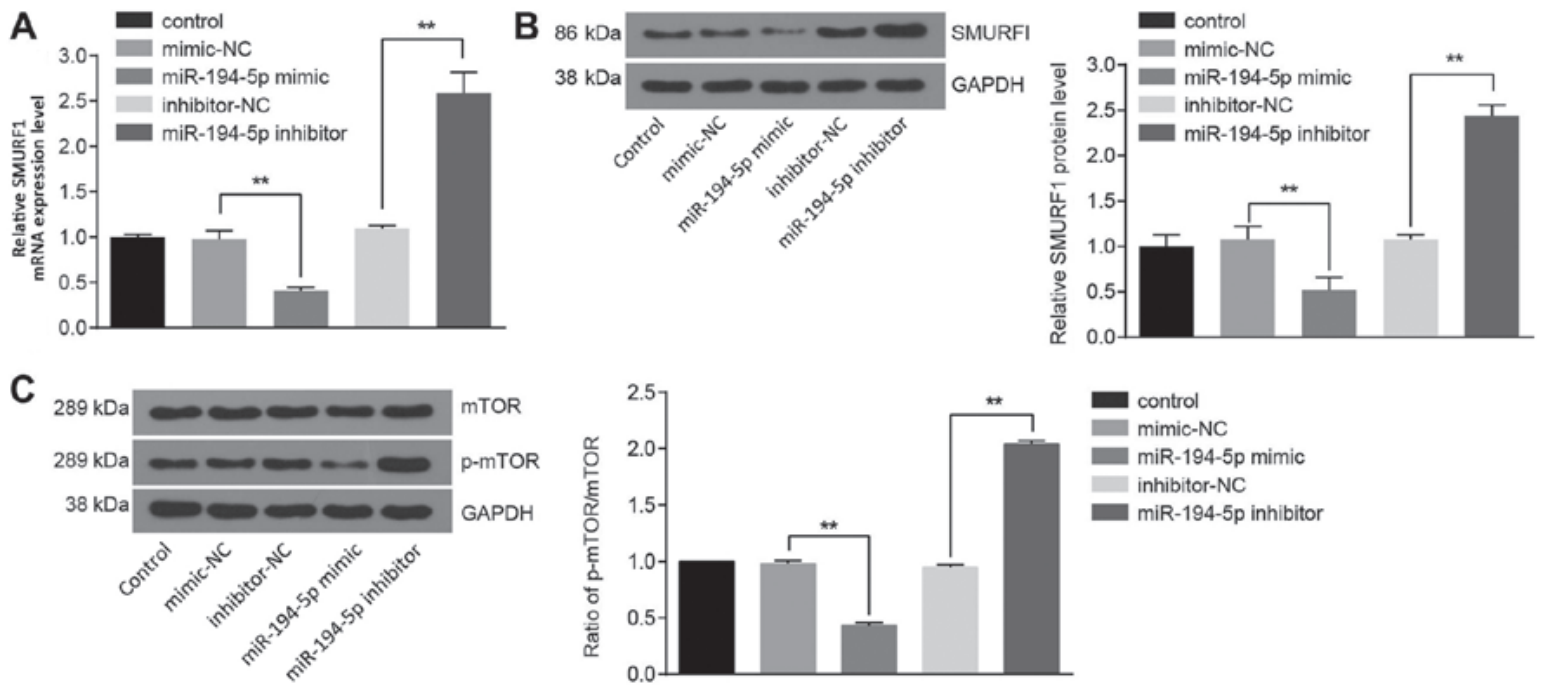

Figure 5. miR-194-5p suppresses the expression of SMURF1 and inhibits mTOR signaling pathway activation. (A) SMURF1 mRNA expression levels in FaDu cells transfected with miR-194-5p mimics or inhibitors were detected by reverse transcription-quantitative polymerase chain reaction. (B) SMURF1 protein expression levels in transfected FaDu cells were detected by western blot analysis. (C) mTOR protein expression and the extent of mTOR phosphorylation in transfected FaDu cells were examined by western blot analysis. Experiments were repeated three times, and the data are presented as the mean \pm standard deviation; ${ }^{* *} \mathrm{P}<0.01$. miR, microRNA; mTOR, mammalian target of rapamycin; NC, negative control; NS, no statistical significance; $p$, phosphorylated; SMURF1, Smad ubiquitin regulatory factor 1 .

in HPC tissues was $76.67 \%$ (23/30), which was significantly higher than that in the adjacent tissues $(16.67 \% ; 5 / 30 ; \mathrm{P}<0.01)$. The results of RT-qPCR (Fig. 4E) and western blot analysis (Fig. 4F) also revealed that the mRNA and protein expression levels, respectively, of SMURF1 were upregulated in HPC tissues compared with adjacent tissues.

Upregulated miR-194-5p inhibits SMURF1 and mTOR signaling pathway activation. mRNA and protein expression levels of SMURF1 and mTOR were examined, as well as the extent of mTOR phosphorylation. Compared with the cells transfected with mimics-NC, the mRNA and protein expression levels of SMURF1 $(\mathrm{P}<0.01$; Fig. 5A and $\mathrm{B}$, respectively), the ratio of p-mTOR to total mTOR in the cells treated with miR-194-5p mimics were significantly decreased $(\mathrm{P}<0.01$; Fig. 5C). However, the mRNA and protein expression levels of SMURF1 ( $\mathrm{P}<0.01$; Fig. 5A and B, respectively), the protein expression of mTOR and the extent of mTOR 
A
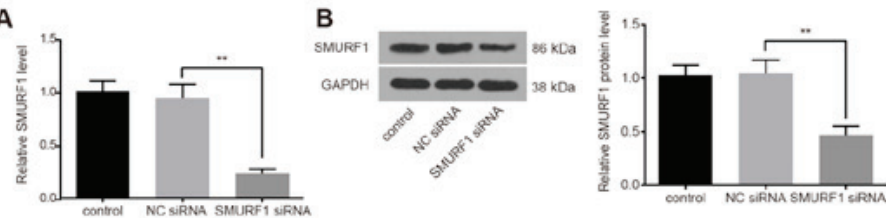

C
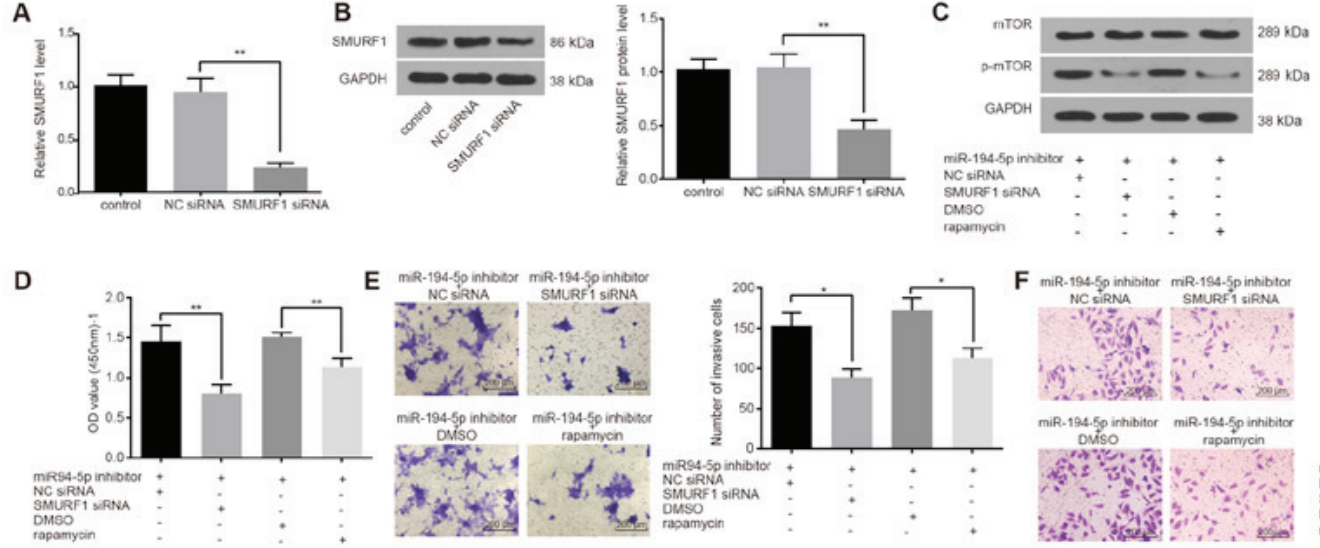

E
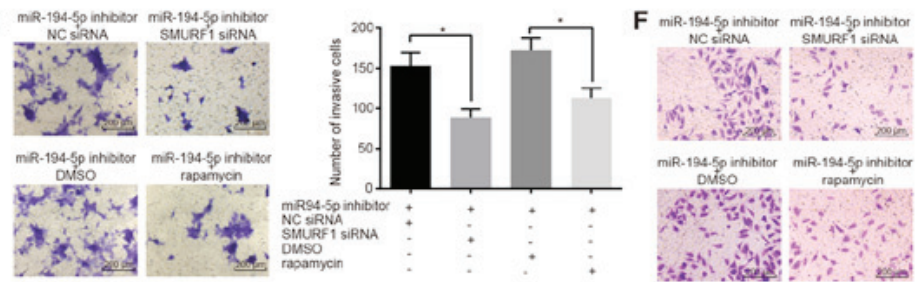

H
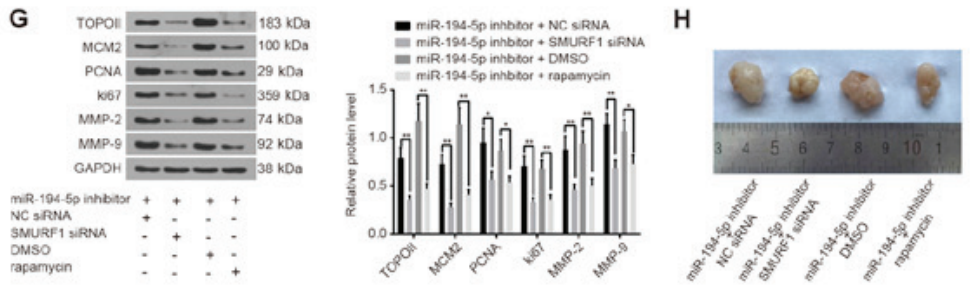

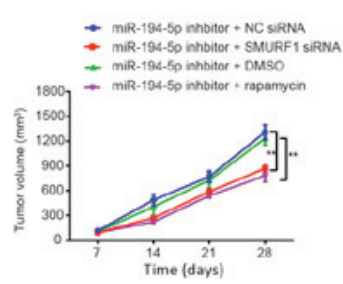

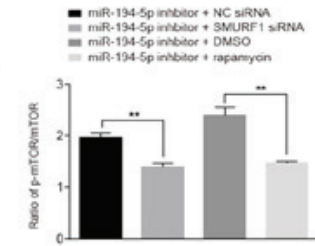
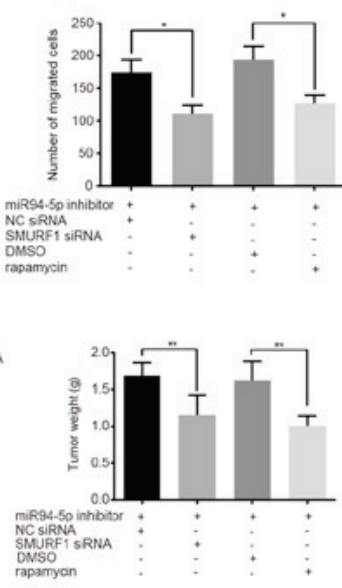

Figure 6. miR-194-5p inactivates the mTOR signaling pathway by targeting SMURF1 to restrain the viability, invasion and migration of FaDu cells, and to inhibit tumor growth. (A and B) SMURF1-siRNA transfection successfully reduced SMURF1 (A) mRNA and (B) protein expression, as detected by reverse transcription-quantitative polymerase chain reaction and western blot analysis, respectively. (C) Protein expression levels of mTOR and the extent of mTOR phosphorylation in transfected FaDu cells were detected by western blot analysis. (D) Viability of FaDu cells at $24 \mathrm{~h}$ post-transfection was determined by Cell Counting Kit-8 assay. (E and F) Invasive and migratory abilities of FaDu cells at 24 h post-transfection detected by (E) Matrigel and (F) Transwell assays. (G) Protein expression levels of proliferation-related (TOPO II, MCM2, PCNA and Ki67) and invasion-related (MMP-2 and MMP-9) factors in transfected FaDu cells were detected by western blot analysis. $(\mathrm{H})$ Nude mice were injected with cells treated with miR-194-5p inhibitor and co-treated with SMURF1-siRNA or rapamycin, and tumor volumes and weights were examined. Experiments were repeated three times, and data are presented as the mean \pm standard deviation; ${ }^{*} \mathrm{P}<0.05 ;{ }^{* *} \mathrm{P}<0.01$. MCM2, minichromosome maintenance 2; miR, microRNA; MMP, matrix metalloproteinase; mTOR, mammalian target of rapamycin; NC, negative control; NS, no statistical significance; p, phosphorylated; PCNA, proliferating cell nuclear antigen; siRNA, small interfering RNA; SMURF1, Smad ubiquitin regulatory factor 1; TOPO II, topoisomerase II.

phosphorylation significantly increased in cells treated with miR-194-5p inhibitor ( $\mathrm{P}<0.01$; Fig. 5C). These data indicated that miR-194-5p may downregulate SMURF1 expression and may block the mTOR signaling pathway.

miR-194-5p inactivates the mTOR signaling pathway by targeting SMURF1 to suppress FaDu cell viability, migration and invasion and to inhibit tumor growth. The regulatory mechanisms of miR-194-5p in HPC and the involvement of SMURF1 and the mTOR signaling pathway were examined. First, the mRNA and protein expression levels of SMURF1 were detected to determine the siRNA-mediated silencing efficiency following SMURF1-siRNA transfection into FaDu cells. Compared with the NC siRNA group, the mRNA and protein expression levels of SMURF1 in the SMURF1-siRNA group were decreased by 75 and 55\%, respectively (Fig. 6A and B, respectively), which indicated that the silencing efficiency was good and that SMURF1-siRNA could be used for subsequent experiments. FaDu cells were treated with miR-194-5p inhibitor and either co-transfected with SMURF1-siRNA or treated with the mTOR inhibitor rapamycin, and the ratio of p-mTOR to total mTOR were evaluated. The protein expression levels of mTOR and the extent of mTOR phosphorylation were significantly decreased in the miR-194-5p inhibitor + SMURF1 siRNA group compared with the miR-194-5p inhibitor + NC siRNA group $(\mathrm{P}<0.01$; Fig. $6 \mathrm{C})$; similar results were observed in the miR-194-5p inhibitor + rapamycin group compared with the
miR-194-5p inhibitor + DMSO group ( $<<0.01$; Fig. 6C). These results suggested that SMURF1 silencing may inactivate the mTOR signaling pathway.

CCK-8, Matrigel and Transwell assays were used to explore the effects of SMURF1 silencing and rapamycin on the viability, invasion and migration of FaDu cells, respectively. Compared with cells treated with miR-194-5p inhibitor alone, the viability, invasion and migration of FaDu cells in the miR-194-5p inhibitor + SMURF1 siRNA and in the miR-194-5p inhibitor + rapamycin groups were significantly inhibited (all $\mathrm{P}<0.05$; Fig. 6D-F).

Western blot analysis was conducted to detect the protein expression levels of proliferation- and invasion-related factors (Fig. 6G). Compared with cells treated with miR-194-5p inhibitor only, the expression levels of the proliferation-related factors TOPO II, MCM2, PCNA and Ki67, and of the invasion-related factors MMP-2 and MMP-9 were significantly decreased in the miR-194-5p inhibitor + SMURF1 siRNA group and in the miR-194-5p inhibitor + rapamycin group $(\mathrm{P}<0.05$ or $\mathrm{P}<0.01$; Fig. $6 \mathrm{G})$. These results suggested that SMURF1 silencing and rapamycin treatment were able to antagonize the miR-194-5p-inhibitor-induced upregulation of mTOR signaling pathway activation on the viability, invasion and migration of FaDu cells and that miR-194-5p may inactivate the mTOR signaling pathway by targeting SMURF1.

A tumorigenicity assay in nude mice was performed to further confirm whether SMURF1 and the mTOR 


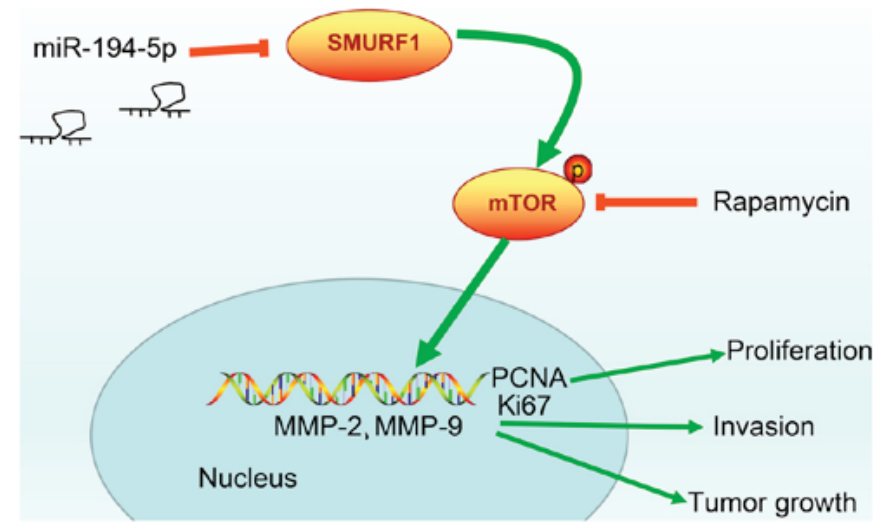

Figure 7. Molecular mechanism of miR-194-5p in HPC involves the mTOR signaling pathway by targeting SMURF1. miR-194-5p inactivates mTOR signaling pathway to downregulate the proliferation-related genes, PCNA and Ki67, and to inhibit invasion-related factors MMP-2 and MMP-9, thereby inhibiting HPC cell proliferation, invasion and tumor growth. HPC, hypopharyngeal carcinoma; MCM2, minichromosome maintenance 2; miR, microRNA; mTOR, mammalian target of rapamycin; MMP, matrix metalloproteinase; PCNA, proliferating cell nuclear antigen; SMURF1, Smad ubiquitin regulatory factor 1 ; TOPO II, topoisomerase II

signaling pathway were regulated by miR-194-5p (Fig. 6H). Compared with the Control nude mice transplanted with miR-194-5p inhibitor + NC siRNA-treated or miR-194-5p inhibitor + DMSO-treated cells, tumor growth in the nude mice transplanted with miR-194-5p inhibitor + SMURF1-siRNA-treated or miR-194-5p inhibitor + rapamycin-treated cells was inhibited and the weight of the tumors decreased significantly at day 28 post-transplantation (all $\mathrm{P}<0.01)$. These in vivo experiments demonstrated that SMURF1 silencing or rapamycin inhibition of the mTOR signaling pathway inhibited tumor growth and further verified that miR-194-5p may inactivate the mTOR signaling pathway by targeting SMURF1.

\section{Discussion}

The prognosis of patients with HPC is often unsatisfactory owing to perioperative complications, even though many of these patients have undergone surgical excision following definitive chemoradiotherapy (18). Consequently, it is of vital importance to study other viable therapeutic strategies to prevent the increasingly prevailing HPC. The results of the present study suggested that miR-194-5p may serve as an anti-oncogene in HPC through its effects on the SMURF1 expression and the mTOR signaling pathway. Consequently, the present study demonstrated that upregulated miR-194-5p may be able to disrupt HPC cell proliferation and metastasis by suppressing SMURF1 and inactivating the mTOR signaling pathway.

In the present study, low expression of miR-194-5p was observed in HPC tissues, and increasing its expression led to the inhibition of cancer cell viability, migration and invasion in vitro. It has been demonstrated previously that miRNAs are aberrantly expressed in various cancers and act as 'tumor suppressor genes' or 'oncogenes' in carcinogenesis (19). Notably, miR-194-5p has been proposed as a tumor suppressor; for example, in glioblastoma multiforme, miR-194-5p inhibits cell growth and promotes apoptosis, thus indicating a suppressive role in this tumor $(20,21)$. Another study suggested that miR-194-5p leads to the downregulation of Bcl-2-associated transcription factor 1, a nuclear protein that binds to Bcl-related proteins, to improvecell differentiation(22). miR-194 was also reported to be expressed at a low level in liver cancer, and its upregulation contributed to the inhibition of cell metastasis in mice (23). All these previous studies have indicated the role of miR-194-5p upregulation in cancer progression. Results from the present study demonstrated that the viability, migration and invasion of the HPC cell line FaDu are suppressed by overexpression of miR-194-5p, which indicated a tumor-suppressive role of miR-194-5p. In addition, it was demonstrated that overexpression of miR-194-5p decreased the protein expression of TOPO II, MCM2, PCNA and Ki67, as well as MMP-2 and MMP-9, in FaDu cells. PCNA and Ki67 are commonly applied as markers for cell proliferation assessment (24). Regarding the role of TOPO II and MCM2 in cell division, those two factors were also considered as markers of cell proliferation or malignant transformation $(25,26)$. These data suggested that miR-194-5p may provide a therapeutic target for HPC.

Results from the present study also revealed that SMURF1 expression was upregulated in HPC tissues. As previously reported, the degradation of ubiquitin-dependent protein is correlated with various biological processes; for example, SMURF1 has been found to be involved in cancer progression (27). Another study reported that the knock down of SMURF1 resulted in a restoration of bone morphogenetic protein signaling and enhanced the induction of differentiation of cancer stem cell-like cells, which may reduce drug resistance and recurrence of head and neck squamous cell carcinoma (11). The present study also demonstrated that FaDu cells transfected with miR-194-5p mimics exhibited reduced proliferation and migration, and miR-194-5p was confirmed to target SMURF1. The gene expression profile of a cell is determined by post-transcriptional processing of mRNA transcripts (28). In the present study, luciferase activity detection verified that miR-194-5p negatively regulated the expression of SMURF1. The results also revealed that SMURF1 silencing blocked the mTOR signaling pathway and prevented HPC progression. Another study also reported that mTOR-inhibiting therapy provided a novel therapeutic approach in patients diagnosed with esophageal squamous cell carcinoma (29). High levels of mTOR phosphorylation were reported to be associated with poor prognosis in esophageal squamous cell carcinoma, indicating that mTOR inhibition may function as a target for the treatment of this disease (30). As previously reported, miR-99a and miR-100 suppress cell proliferation by inhibiting the mTOR signaling pathway in human esophageal squamous cell carcinoma (31). Another study reported that high SMURF1 expression levels may result in enhanced activation of the PI3K/AKT/mTOR signaling pathway (31). Partly in line with the present study results, Tao et al demonstrated that SMURF1 knockdown significantly suppressed the migration, invasion and tumor growth of gastric cancer, whereas it was involved with a mechanism involving the PI3K/Akt pathway and the SMURF1/DABIP axis (32). These results indicated that miR-194-5p may hinder HPC progression through inhibiting the mTOR signaling pathway by downregulating SMURF1. 
In summary, the present study data provided evidence that upregulation of miR-194-5p may effectively repress cell proliferation and invasion in HPC, whereas the expression of SMURF1 is downregulated and the mTOR signaling pathway is inactivated (Fig. 7). These results indicate a potential regulatory mechanism of miR-194-5p targeting SMURF1 to block the mTOR signaling pathway. Therefore, it is suggested that the miR-194-5p/SMURF1/mTOR axis may serve as an attractive therapeutic target for HPC treatment, and miR-194-5p mimic treatment might be a better alternative to the known treatment with rapamycin. However, the results of this study were obtained using a sample size. Therefore, further studies on the more detailed mechanisms as well as the effectiveness and safety of miR-194-5p in the treatment of this fatal malignancy should be analyzed with a larger sample size.

\section{Acknowledgements}

Not applicable.

\section{Funding}

This study was supported by The Natural Science Foundation of Liaoning Province (grant nos. 20170541050 and 20170541026).

\section{Availability of data and materials}

The data sets used and/or analyzed during the current study are available from the corresponding author on reasonable request.

\section{Authors' contributions}

SX and LH conceived and designed the experiments, and wrote the paper. NY and YW obtained the and validated the results. NZ and XJJ reviewed the results and discussions. SX, LH and NY performed the statistical analysis and prepared figures. All authors had final approval of the submitted and published versions.

\section{Ethics approval and consent to participate}

The present study was approved by the Ethics Committee of The First Hospital of China Medical University (Shenyang, China), and written informed consent was obtained from each participant prior to enrollment. The animal experiments were conducted with approval of the Animal Ethics Committee of The First Hospital of China Medical University, and all animal experimental procedures conformed to the provisions of the Guide for the Care and Use of Laboratory Animals by US National Institutes of Health.

\section{Patient consent for publication}

Not applicable.

\section{Competing interests}

The authors declare that they have no competing interests.

\section{References}

1. Zhang Y, Wang B, Chen X, Li W and Dong P: AGO2 involves the malignant phenotypes and FAK/PI3K/AKT signaling pathway in hypopharyngeal-derived FaDu cells. Oncotarget 8: 54735-54746, 2017.

2. Wu P, Wu H, Tang Y, Luo S, Fang X, Xie C, He J, Zhao S, Wang X, $\mathrm{Xu}$ J, et al: Whole-exome sequencing reveals novel mutations and epigenetic regulation in hypopharyngeal carcinoma. Oncotarget 8: 85326-85340, 2017.

3. Chan JY and Wei WI: Current management strategy of hypopharyngeal carcinoma. Auris Nasus Larynx 40: 2-6, 2013.

4. Zhang Y, Cong L, He J, Wang Y, Zou Y, Yang Z, Hu Y, Zhang S, and He X: Photothermal treatment with EGFRmAb-AuNPs induces apoptosis in hypopharyngeal carcinoma cells via $\mathrm{PI} 3 \mathrm{~K} / \mathrm{AKT} / \mathrm{mTOR}$ and DNA damage response pathways. Acta Biochim Biophys Sin (Shanghai) 50: 567-578, 2018.

5. Orenes-Piñero E, Montoro-García S, Patel JV, Valdés M, Marín F and Lip GY: Role of microRNAs in cardiac remodelling: New insights and future perspectives. Int J Cardiol 167: 1651-1659, 2013.

6. Nohata N, Hanazawa T, Kinoshita T, Okamoto Y and Seki N: MicroRNAs function as tumor suppressors or oncogenes: Aberrant expression of microRNAs in head and neck squamous cell carcinoma. Auris Nasus Larynx 40: 143-149, 2013.

7. Han C, Shen JK, Hornicek FJ, Kan Q and Duan Z: Regulation of microRNA-1 (miR-1) expression in human cancer. Biochim Biophys Acta Gene Regul Mech 1860: 227-232, 2017.

8. Choi JS, Nam MH, Yoon SY and Kang SH: MicroRNA-194-5p could serve as a diagnostic and prognostic biomarker in myelodysplastic syndromes. Leuk Res 39: 763-768, 2015.

9. Yumioka T, Osaki M, Sasaki R, Yamaguchi N, Onuma K, Iwamoto H, Morizane S, Honda M, Takenaka A and Okada F: Lysosome-associated membrane protein 2 (LAMP-2) expression induced by miR-194-5p downregulation contributes to sunitinib resistance in human renal cell carcinoma cells. Oncol Lett 15: 893-900, 2018.

10. Wei R, Li B, Guo J, Li M, Zhu R, Yang X and Gao R: Smurf1 targets Securin for ubiquitin-dependent degradation and regulates the metaphase-to-anaphase transition. Cell Signal 38: 60-66, 2017.

11. Khammanivong A, Gopalakrishnan R and Dickerson EB: SMURF1 silencing diminishes a CD44-high cancer stem cell-like population in head and neck squamous cell carcinoma. Mol Cancer 13: 260, 2014.

12. Ke M, Mo L, Li W, Zhang X, Li F and Yu H: Ubiquitin ligase SMURF1 functions as a prognostic marker and promotes growth and metastasis of clear cell renal cell carcinoma. FEBS Open Bio 7: 577-586, 2017.

13. Guertin DA and Sabatini DM: Defining the role of mTOR in cancer. Cancer Cell 12: 9-22, 2007.

14. Hou G, Xue L, Lu Z, Fan T, Tian F and Xue Y: An activated $\mathrm{mTOR} / \mathrm{p} 70$ S6K signaling pathway in esophageal squamous cell carcinoma cell lines and inhibition of the pathway by rapamycin and siRNA against mTOR. Cancer Lett 253: 236-248, 2007.

15. USDA Animal Care: Act Animal Welfare and Regulations Animal Welfare. United States Department of Agriculture, Washington, DC, 2003.

16. Ito K, Liu Q, Salto-Tellez M, Yano T, Tada K, Ida H, Huang C, Shah N, Inoue M, Rajnakova A, et al: RUNX3, a novel tumor suppressor, is frequently inactivated in gastric cancer by protein mislocalization. Cancer Res 65: 7743-7750, 2005.

17. Livak KJ and Schmittgen TD: Analysis of relative gene expression data using real-time quantitative PCR and the $2(-\Delta \Delta \mathrm{C}(\mathrm{T}))$ method. Methods 25: 402-408, 2001.

18. Kadota H, Fukushima J, Nakashima T, Kumamoto Y, Yoshida S, Yasumatsu R, Shiratsuchi H, Morita M and Komume S: Comparison of salvage and planned pharyngolaryngectomy with jejunal transfer for hypopharyngeal carcinoma after chemoradiotherapy. Laryngoscope 120: 1103-1108, 2010.

19. Li Q, Qiu XM, Li QH, Wang XY, Li L, Xu M, Dong M and Xiao YB: MicroRNA-424 may function as a tumor suppressor in endometrial carcinoma cells by targeting E2F7. Oncol Rep 33: 2354-2360, 2015.

20. Zhang Z, Lei B, Wu H, Zhang X and Zheng N: Tumor suppressive role of miR-194-5p in glioblastoma multiforme. Mol Med Rep 16: 9317-9322, 2017.

21. Su R, Cao S, Ma J, Liu Y, Liu X, Zheng J, Chen J, Liu L, Cai H, Li Z, et al: Knockdown of SOX2OT inhibits the malignant biological behaviors of glioblastoma stem cells via up-regulating the expression of miR-194-5p and miR-122. Mol Cancer 16: 171, 2017. 
22. Dell'Aversana C, Giorgio C, D'Amato L, Lania G, Matarese F, Saeed S, Di Costanzo A, Belsito Petrizzi V, Ingenito C, Martens JHA, et al: miR-194-5p/BCLAF1 deregulation in AML tumorigenesis. Leukemia 31: 2315-2325, 2017.

23. Meng Z, Fu X, Chen X, Zeng S, Tian Y, Jove R, Xu R and Huang W: miR-194 is a marker of hepatic epithelial cells and suppresses metastasis of liver cancer cells in mice. Hepatology 52: 2148-2157, 2010

24. Bologna-Molina R, Mosqueda-Taylor A, Molina-Frechero N, Mori-Estevez AD and Sánchez-Acuña G: Comparison of the value of PCNA and Ki-67 as markers of cell proliferation in ameloblastic tumors. Med Oral Patol Oral Cir Bucal 18: e174-e179, 2013

25. Stromar IK and Jakic-Razumovic J: The value of immunohistochemical determination of topoisomerase II $\alpha$ and Ki67 as markers of cell proliferation and malignant transformation in colonic mucosa. Appl Immunohistochem Mol Morphol 22: 524-529, 2014.

26. Carreón-Burciaga RG, González-González R, Molina-Frechero N and Bologna-Molina R: Immunoexpression of $\mathrm{Ki}-67, \mathrm{MCM} 2$ and MCM3 in Ameloblastoma and Ameloblastic Carcinoma and Their Correlations with Clinical and Histopathological Patterns. Dis Markers 2015: 683087, 2015.

27. Fukunaga E, Inoue Y, Komiya S, Horiguchi K, Goto K, Saitoh M, Miyazawa K, Koinuma D, Hanyu A and Imamura T: Smurf2 induces ubiquitin-dependent degradation of Smurf1 to prevent migration of breast cancer cells. J Biol Chem 283: 35660-35667, 2008
28. Wigington CP, Jung J, Rye EA, Belauret SL, Philpot AM, Feng Y, Santangelo PJ and Corbett AH: Post-transcriptional regulation of programmed cell death 4 (PDCD4) mRNA by the RNA-binding proteins human antigen $\mathrm{R}(\mathrm{HuR})$ and $\mathrm{T}$-cell intracellular antigen 1 (TIA1). J Biol Chem 290: 3468-3487, 2015.

29. Boone J, Ten Kate FJ, Offerhaus GJ, van Diest PJ, Rinkes IH and van Hillegersberg R: mTOR in squamous cell carcinoma of the oesophagus: A potential target for molecular therapy? J Clin Pathol 61: 909-913, 2008.

30. Hirashima K, Baba Y, Watanabe M, Karashima R, Sato N, Imamura Y, Hiyoshi Y, Nagai Y, Hayashi N, Iyama K, et al: Phosphorylated mTOR expression is associated with poor prognosis for patients with esophageal squamous cell carcinoma. Ann Surg Oncol 17: 2486-2493, 2010.

31. Sun J, Chen Z, Tan X, Zhou F, Tan F, Gao Y, Sun N, Xu X, Shao K and He J: MicroRNA-99a/100 promotes apoptosis by targeting mTOR in human esophageal squamous cell carcinoma. Med Oncol 30: 411, 2013

32. Tao Y, Sun C, Zhang $T$ and Song Y: SMURF1 promotes the proliferation, migration and invasion of gastric cancer cells. Oncol Rep 38: 1806-1814, 2017.

(i) $($ This work is licensed under a Creative Commons Attribution-NonCommercial-NoDerivatives 4.0 International (CC BY-NC-ND 4.0) License. 